\title{
A COMPARISON THEOREM FOR LIE ALGEBRA HOMOLOGY GROUPS
}

\author{
Tim BRATten
}

Let $M$ be a Harish-Chandra module associated to a finite length, admissible representation of real reductive Lie group $G_{0}$. Suppose that $\mathfrak{p}$ is a parabolic subalgebra of the complexified Lie algebra of $G_{0}$ and let $\mathfrak{n} \subset \mathfrak{p}$ be the nil radical of $\mathfrak{p}$. In this paper, motivated by some recent work in the study of zeta functions on locally symmetric spaces, we make a comparison between homological properties of $M$ and homological properties of the minimal globalization of $M$. In particular, if $\mathfrak{p}$ has a real Levi factor, we are able to show that, after conjugating by an element from $G_{0}$, then the n-homology groups of the minimal globalization of $M$ are, in a natural way, the minimal globalizations of the $\mathfrak{n}$-homology groups of $M$.

\section{Introduction.}

Some recent work in the study of zeta functions defined on locally symmetric spaces has focused on comparing the homological properties of a Harish-Chandra module with the homological properties of various global representations associated to that Harish-Chandra module. In particular, one recent paper by U. Bunke and M. Olbrich [3] establishes a comparison theorem for some Lie algebra cohomology groups in order to describe the singularities of the Selberg zeta function. Even more recently, the same authors have extended their work on the comparison theorem [4] by applying a result of Kashiwara and Schmid [14]. On the other hand, during the austral autumn of 1995, A. Deitmar expressed to this author a desire to know a comparison theorem in some generality, in order to obtain some results in his study of zeta functions associated to compact Shimura manifolds [7]. It was this need that provided the motivation for writing down the following argument.

The purpose of this article is to show that a comparison theorem of a very general nature can be deduced from a result in [2] by a series of standard formal reductions. The result in [4] then becomes a special case of our result, although the methods we utilize are quite different and stem from the work of H. Hecht and J. Taylor [12], [13]. 
Specifically, suppose $G_{0}$ is a real reductive group of Harish-Chandra class [8] and let $\mathfrak{g}$ be the complexified Lie algebra of $G_{0}$. Recall that a Borel subalgebra of $\mathfrak{g}$ is a maximal solvable subalgebra and that a parabolic subalgebra is any subalgebra that contains a Borel subalgebra.

Let $\tau: \mathfrak{g} \rightarrow \mathfrak{g}$ denote the conjugation of $\mathfrak{g}$ determined by $G_{0}$ and fix a maximal compact subgroup $K_{0} \subset G_{0}$. We choose a Cartan involution of $G_{0}$ whose fixed point set is $K_{0}$ and let $\theta: \mathfrak{g} \rightarrow \mathfrak{g}$ denote the extension to $\mathfrak{g}$ of the corresponding derivative. A subalgebra of $\mathfrak{g}$ will be called stable if it is invariant under both $\tau$ and $\theta$. A parabolic subalgebra $\mathfrak{p} \subset \mathfrak{g}$ is said to be special of Levi type if it contains a stable Levi factor $\mathfrak{l}$. Such a Levi factor (if it exists) is unique.

Fix a special parabolic subalgebra $\mathfrak{p}$ of Levi type with nil radical $\mathfrak{n}$ and stable Levi factor $\mathfrak{l}$. Let $L_{0}$ be the subgroup of $G_{0}$ that normalizes both $\mathfrak{l}$ and $\mathfrak{n}$. Then $L_{0}$ is a real reductive group of Harish-Chandra class wih complexified Lie algebra $\mathfrak{l}$ and maximal compact subgroup $K_{0} \cap L_{0}$. We refer to $L_{0}$ as the real Levi factor associated to $\mathfrak{p}$.

Let $K$ be the complexification of $K_{0}$. A $\mathfrak{g}$-module $M$ is called a HarishChandra module for $(\mathfrak{g}, K)$ provided $M$ carries a compatible, algebraic [9, Section 2, A1 and A2], $K$ action and provided that the resulting $(\mathfrak{g}, K)$ structure has finite length. Let $K_{L} \subset K$ be the complexification of $K_{0} \cap L_{0}$. Then the Lie algebra homology groups

$$
H_{p}(\mathfrak{n}, M)
$$

are Harish-Chandra modules for $\left(\mathfrak{l}, K_{L}\right)$ [18].

Given a Harish-Chandra module $M$ we let $M_{\min }$ denote its minimal globalization [14], [17]. We remark that the minimal globalization is a canonical and functorially defined topological $G_{0}$-module on a dual nuclear Fréchet $(=d n F)$ space that consists entirely of analytic vectors. As the name suggests, $M_{\min }$ embeds continuously and equivariantly in any topological representation of $G_{0}$, on a complete locally convex space, that contains $M$ as its underlying space of $K_{0}$-finite vectors. Our main result is the following:

Theorem 1. Let $\mathfrak{p}$ be a special parabolic subalgebra of Levi type with corresponding Levi decomposition $\mathfrak{p}=\mathfrak{l}+\mathfrak{n}$ and let $M$ be a Harish-Chandra module for $(\mathfrak{g}, K)$. Fix a $\left(\mathfrak{g}, K_{0}\right)$ equivariant inclusion $M \rightarrow M_{\min }$ onto the $K_{0}$ finite vectors in $M_{\min }$. Let $L_{0}$ denote the real Levi factor associated to $\mathfrak{p}$. (a) For each $p$, the Lie algebra homology groups $H_{p}\left(\mathfrak{n}, M_{\min }\right)$ have naturally defined $d n F$ topologies and continuous $L_{0}$ actions.

(b) The inclusion $M \rightarrow M_{\min }$ determines a $L_{0}$ equivariant topological isomorphism $H_{p}(\mathfrak{n}, M)_{\min } \simeq H_{p}\left(\mathfrak{n}, M_{\min }\right)$.

We remark that the result in [4] establishes the comparison theorem for 
parabolic subalgebras of $\mathfrak{g}$ defined over $\mathbb{R}$ (i.e. any $\mathfrak{p}$ such that $\tau \mathfrak{p}=\mathfrak{p}$ ). A parabolic subalgebra defined over $\mathbb{R}$ is always special of Levi type, since in this case $\mathfrak{p} \cap \theta \mathfrak{p}$ is a stable Levi factor. On the other hand, it is possible to define entire manifolds of parabolic subalgebras of $\mathfrak{g}$ such that each point is conjugate to a special parabolic subalgebra of Levi type, yet none of the points is a parabolic subalgebra defined over $\mathbb{R}$. For example suppose $G_{0}$ is any real reductive group which is not split and consider the full flag manifold $X$ of Borel subalgebras of $\mathfrak{g}$. A result of Matsuki [15] implies that every point in $X$ is $G_{0}$-conjugate to a special point of Levi type, but in this example no Borel subalgebra of $\mathfrak{g}$ is defined over $\mathbb{R}$. In the case of a full flag manifold, the comparison theorem was already shown in [13].

The paper is organized as follows. In the second and third sections we give a proof of the comparison theorem. Our first task is to translate a geometric result established in [2] into the language of $\mathfrak{n}$-homologies using a dictionary which is nearly identical to the well known case of a full flag manifold. Formal considerations and tensoring arguments complete the proof. We then finish the article by rephrasing our result in terms of Lie algebra cohomology and a topological duality.

This paper was motivated by some discussions with Anton Deitmar. The author has also benefited from some helpful discussions with Jorge Vargas. The work for this paper was completed while the author was supported by a post-doctoral position in FaMAF, Universidad Nacional de Córdoba.

\section{Translating the geometric argument.}

Let $\mathfrak{p}=\mathfrak{l}+\mathfrak{n}$ be a Levi decompostion for a parabolic subalgebra of $\mathfrak{g}$ and let $M$ be a $\mathfrak{g}$ module. Let $U(\mathfrak{g})$ and $U(\mathfrak{n})$ denote the enveloping algebras of $\mathfrak{g}$ and $\mathfrak{n}$, respectively. We define the $\mathfrak{n}$-homologies of $M$ to be the derived functors for the right exact functor of coinvariants

$$
H_{0}(\mathfrak{n}, M)=\mathbb{C} \otimes_{U(\mathfrak{n})} M
$$

from the category of $\mathfrak{g}$-modules to the category of $\mathfrak{l}$-modules. Since $U(\mathfrak{g})$ is a free $U(\mathfrak{n})$-module, the derived functors for the coinvariants funtor can be computed using resolutions by free $U(\mathfrak{g})$-modules.

Let $Z(\mathfrak{g})$ denote the center of $U(\mathfrak{g})$. A homomorphism of algebras $\sigma$ : $Z(\mathfrak{g}) \rightarrow \mathbb{C}$ is called an infinitesimal character. Given an infinitesimal character $\sigma$, we let $U_{\sigma}$ denote the corresponding quotient of $U(\mathfrak{g})$ by the ideal generated from the kernel of $\sigma$. Since $U_{\sigma}$ is a free $U(\mathfrak{n})$-module [16] it follows that we can compute the derived functors for the coinvariants of a $U_{\sigma}$ module using resolutions by free $U_{\sigma}$-modules. 
Let $Z(\mathfrak{l})$ denote the center of $U(\mathfrak{l})$. When $M$ is a $U_{\sigma}$ module (or more generally a $Z(\mathfrak{g})$ finite module) then the homology groups $H_{p}(\mathfrak{n}, M)$ are finite direct sums of generalized $Z(\mathfrak{l})$ eigenspaces [5], [18]. In fact, the HarishChandra maps make the relation between the character $\sigma$ and these generalized eigenvalues explicit. We adopt the notations used in [2], [12]. Specifically, let $\mathfrak{h}$ be the universal Cartan subalgebra of $\mathfrak{g}$. There are unnormalized Harish-Chandra maps: $Z(\mathfrak{g}) \rightarrow U(\mathfrak{h})$ and $Z(\mathfrak{l}) \rightarrow U(\mathfrak{h})$. In addition, there is an injection of algebras $Z(\mathfrak{g}) \rightarrow Z(\mathfrak{l})$ uniquely determined by the requirement that the following diagram commute:

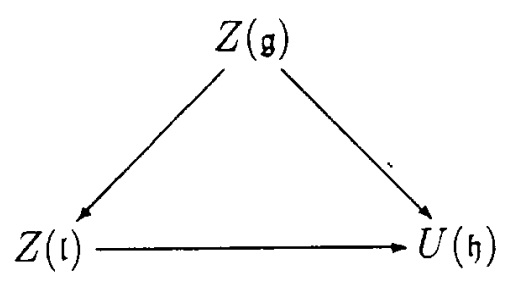

Let $\Sigma \subset \mathfrak{h}^{*}$ be the universal root system, let $W$ denote the Weyl group of $\Sigma$ and let $W_{\mathrm{l}} \subset W$ be the Weyl group corresponding to the Levi factor $\mathfrak{l} \subset \mathfrak{p}$. A fundamental result of Harish-Chandra uses the above morphisms to define identifications of the characters of $Z(\mathfrak{g})$ (also of $Z(\mathfrak{l})$ ) with $W$ orbits (repectively $W_{\mathfrak{l}}$ orbits) in $\mathfrak{h}^{*}$. In particular, let $\Sigma^{+} \subset \Sigma$ be the set of positive roots [2], [12] and let $\rho$ be one half the sum of the elements in $\Sigma^{+}$. Then $\lambda \in \mathfrak{h}^{*}$ is identified with a character of $Z(\mathfrak{l})$ via the map:

$$
Z(\mathfrak{l}) \rightarrow U(\mathfrak{h}) \stackrel{\lambda+\rho}{\longrightarrow} \mathbb{C} .
$$

Suppose that $\sigma=W \cdot \lambda$. Then $\sigma$ is called regular provided $\check{\alpha}(\lambda)$ is not zero for each $\alpha \in \Sigma$. Let $J_{\sigma} \subset Z(\mathfrak{g})$ be the kernel of $\sigma$ and identify $J_{\sigma}$ with a subset of $Z(\mathfrak{l})$ via the morphism $Z(\mathfrak{g}) \rightarrow Z(\mathfrak{l})$. Let $V_{\sigma}^{\mathfrak{l}}$ denote the quotient of $Z(\mathfrak{l})$ by the ideal generated from $J_{\sigma}$.

Lemma 1. $\quad V_{\sigma}^{\mathfrak{l}}$ is a finite dimensional $Z(\mathfrak{l})$-module which is semisimple when $\sigma$ is regular.

Proof. Identify $Z(\mathfrak{g})$ and $Z(\mathfrak{l})$ with subalgebras of $U(\mathfrak{h})$ via the HarishChandra maps. Let $V_{\sigma}^{\mathfrak{h}}$ denote the quotient of $U(\mathfrak{h})$ by the ideal generated from $J_{\sigma}$. Then it is well known that $V_{\sigma}^{\mathfrak{h}}$ is a finite dimensional vector space which is semisimple as an $\mathfrak{h}$-module (and hence as a $Z(\mathfrak{l})$-module) when $\sigma$ is regular [16]. Therefore it is sufficient to see that the morphism $V_{\sigma}^{\mathfrak{l}} \rightarrow V_{\sigma}^{\mathfrak{h}}$ of $Z(\mathfrak{l})$ modules is injective. But this last point is a straightforward application of the identification of $Z(\mathfrak{l})$ with the $W_{\mathfrak{l}}$ invariants in $U(\mathfrak{h})$. 
If $V$ is an $\mathfrak{l}$ module and if $\lambda \in \mathfrak{h}^{*}$ let $V_{\lambda}$ denote the $Z(\mathfrak{l})$ eigenspace corresponding to the infinitesimal character $\chi_{\lambda}=W_{\mathfrak{l}} \cdot \lambda$.

Lemma 2. Suppose that $\sigma=W \cdot \lambda$ is a regular infinitesimal character for $Z(\mathfrak{g})$. Then $F(M)=H_{0}(\mathfrak{n}, M)_{\lambda}$ defines a right exact functor from the category of $U_{\sigma}$ modules to the category of $U(\mathfrak{l})$ modules with derived functors $L_{p} F(M)$ isomorphic to $H_{p}(\mathfrak{n}, M)_{\lambda}$.

Proof. If $M$ is any $U_{\sigma}$ module then there is a surjection $V_{\sigma}^{\mathfrak{l}} \otimes H_{0}(\mathfrak{n}, M) \rightarrow$ $H_{0}(\mathfrak{n}, M) \rightarrow 0$ of $Z(\mathfrak{l})$ modules. By the previous lemma $V_{\sigma}^{\mathfrak{l}}$ is a finite direct sum of $Z(\mathfrak{l})$ eigenspaces so that $H_{0}(\mathfrak{n}, M)$ is too. Hence $H_{0}(\mathfrak{n}, M)$ belongs to the category of $U(\mathfrak{l})$ modules which are finite direct sums of $Z(\mathfrak{l})$ eigenspaces. The result follows since the functor $V \mapsto V_{\lambda}$ is exact on this last category.

We now introduce the geometrical data. Let $\operatorname{Int}(\mathfrak{g})$ be the complex semisimple adjoint group of $\mathfrak{g}$ and let $Y$ be the quotient of $\operatorname{Int}(\mathfrak{g})$ by the normalizer of $\mathfrak{p}: Y$ is nothing but the flag manifold consisting of all $\operatorname{Int}(\mathfrak{g})$ conjugates of $\mathfrak{p}$. In particular, $Y$ is a smooth projective variety. Let $y \in Y$ denote the point in $Y$ corresponding to the parabolic subalgebra $\mathfrak{p}$ and let $\mathcal{O}_{Y}$ be the sheaf of regular functions on $Y$.

For $\lambda \in \mathfrak{h}^{*}$ let $\mathcal{A}_{\lambda}$ denote the corresponding generalized twisted sheaf of differential operators on $Y$ [2, Notation as in Section 3] [6, Definition 4.3]. Let $X$ denote full flag manifold for $\mathfrak{g}$ (this is the projective variety of Borel subalgebras of $\mathfrak{g})$. Then the sheaf $\mathcal{A}_{\lambda}$ is nothing but the direct image of a twisted sheaf of differential operators $\mathcal{D}_{\lambda}$ (with regular coefficients) under the standard projection $\pi: X \rightarrow Y$. If $\sigma=W \cdot \lambda$ is the character of $Z(\mathfrak{g})$ corresponding to $\lambda$ then $U_{\sigma}=\Gamma\left(Y, \mathcal{A}_{\lambda}\right)$ [1], [16]. Suppose $M$ is a $U_{\sigma}$-module. The localization of $M$ to $Y$ is the quasicoherent sheaf of $\mathcal{A}_{\lambda}$ modules defined by:

$$
\Delta_{\lambda}(M)=\mathcal{A}_{\lambda} \otimes_{U_{\sigma}} M
$$

On the other hand, suppose $\mathcal{M}$ is a sheaf of $\mathcal{A}_{\lambda}$ modules and let $\mathcal{M}_{y}$ denote the stalk of $\mathcal{M}$ over the point $y \in Y$. The geometric fiber of $\mathcal{M}$ at $y$ is defined by:

$$
T_{y}(\mathcal{M})=\mathbb{C} \otimes_{\mathcal{O}_{Y} y} \mathcal{M}_{y}
$$

Because $\exp \left(\operatorname{ad}_{L}\right) \in \operatorname{Int}(\mathfrak{g})$ stablizes $y$ for each $L \in \mathfrak{l}$ it follows that the morphism $U(\mathfrak{l}) \rightarrow \Gamma\left(Y, \mathcal{A}_{\lambda}\right)$ determines a left $\mathfrak{l}$ action on $T_{y}(\mathcal{M})$.

Proposition 1. Suppose $\sigma=W \cdot \lambda$ is a is a regular infinitesimal character and suppose $M$ is a $U_{\sigma}$-module. Then $H_{0}(\mathfrak{n}, M)_{\lambda}$ and $T_{y} \circ \Delta_{\lambda}(M)$ define 
isomorphic functors from the category of $U_{\sigma}$ modules to the category of $U(\mathfrak{l})$ modules.

Proof. According to Lemma 2, $H_{0}(\mathfrak{n}, M)$ is a finite direct sum of $Z(\mathfrak{l})$ eigenspaces. Hence, the natural isomorphism: $H_{0}\left(\mathfrak{n}, U_{\sigma}\right) \otimes_{U_{\sigma}} M \simeq H_{0}(\mathfrak{n}, M)$ descends to an isomorphism: $H_{0}\left(\mathfrak{n}, U_{\sigma}\right)_{\lambda} \otimes_{U_{\sigma}} M \simeq H_{0}(\mathfrak{n}, M)_{\lambda}$. This means it suffices to show that $H_{0}\left(\mathfrak{n}, U_{\sigma}\right)_{\lambda}$ and $T_{y}\left(\mathcal{A}_{\lambda}\right)$ are isomorphic as $(\mathfrak{l}, \mathfrak{g})$ bimodules.

According to [6, Proposition 4.5], [12, Proposition 6.1] there is an isomorphism of $(\mathfrak{l}, \mathfrak{g})$ bimodules: $\mathbb{C}_{\lambda} \otimes_{Z(\mathfrak{l})} H_{0}(\mathfrak{n}, U(\mathfrak{g})) \simeq T_{y}\left(\mathcal{A}_{\lambda}\right)$. Let $J_{\sigma} \subset Z(\mathfrak{g})$ be the kernel of $\sigma$ and consider the exact sequence:

$$
\mathbb{C}_{\lambda} \otimes_{Z(\mathfrak{l})} H_{0}\left(\mathfrak{n}, J_{\sigma} U(\mathfrak{g})\right) \rightarrow \mathbb{C}_{\lambda} \otimes_{Z(\mathfrak{l})} H_{0}(\mathfrak{n}, U(\mathfrak{g})) \rightarrow \mathbb{C}_{\lambda} \otimes_{Z(\mathfrak{l})} H_{0}\left(\mathfrak{n}, U_{\sigma}\right) \rightarrow 0 .
$$

Another application of Lemma 2 shows that this last space is nothing but $H_{0}\left(\mathfrak{n}, U_{\sigma}\right)_{\lambda}$. By considering the morphism $Z(\mathfrak{g}) \rightarrow Z(\mathfrak{l})$ one immediately sees that $\mathbb{C}_{\lambda} \otimes_{Z(\mathfrak{l})} H_{0}\left(\mathfrak{n}, J_{\sigma} U(\mathfrak{g})\right)$ is zero.

An element $\lambda \in \mathfrak{h}^{*}$ is called antidominant provided $\check{\alpha}(\lambda)$ is not a positive integer for each $\alpha \in \Sigma^{+}$. We say $\lambda$ is antidominant for $Y$ provided $\lambda$ is $W_{\mathfrak{r}}$ conjugate to something antidominant.

Lemma 3. Assume $\lambda$ is regular and antidominant for $Y$ and suppose $M$ is a Harish-Chandra module for $(\mathfrak{g}, K)$ with an infinitesimal character. Let $M_{\min }$ denote the minimal globalization of $M$. Assume $\mathfrak{p}$ is a special parabolic subalgebra of Levi type with corresponding Levi decomposition $\mathfrak{p}=\mathfrak{l}+\mathfrak{n}$. Let $L_{0}$ be the real Levi factor associated to $\mathfrak{p}$.

(a) The $\mathfrak{l}$ module $H_{p}\left(\mathfrak{n}, M_{\min }\right)_{\lambda}$ has a naturally defined $d n F$ topology and a continuous $L_{0}$ action.

(b) Any $\left(\mathfrak{g}, K_{0}\right)$ equivariant inclusion $M \rightarrow M_{\min }$ onto the $K_{0}$ finite vectors in $M_{\min }$ determines an $L_{0}$ equivariant isomorphism $\left(H_{p}(\mathfrak{n}, M)_{\lambda}\right)_{\min } \simeq$ $H_{p}\left(\mathfrak{n}, M_{\min }\right)_{\lambda}$.

Proof. Suppose $M$ (and therefore $M_{\min }$ as well) has infinitesimal character $\sigma$. According to the Casselman-Osborne Lemma [5] the homology groups $H_{p}(\mathfrak{n}, M)_{\lambda}$ and $H_{p}\left(\mathfrak{n}, M_{\min }\right)_{\lambda}$ will both vanish for all $p$ unless $\sigma=W \cdot \lambda$. Therefore we assume $\sigma=W \cdot \lambda$.

Let $F .(M)$ and $F .\left(M_{\min }\right)$ be the Hochschild resolutions of $M$ and $M_{\min }$ respectively (these are just canonical resolutions by free $U_{\sigma}$ modules) [2], [11]. In particular:

$$
F_{p}\left(M_{\min }\right)=\otimes^{p+1} U_{\sigma} \otimes M_{\min } .
$$

Since $U_{\sigma}$ is a $d n F$ space with a countable basis it follows that $F_{p}\left(M_{\min }\right)$ is a $d n F U_{\sigma}$ module [12]. Via the tensor product of the adjoint action on $U_{\sigma}$ with 
the action on $M_{\min }$ one obtains a continuous $G_{0}$-action on $F_{p}\left(M_{\min }\right)$. This action is not compatible with the left $\mathfrak{g}$ action, nevertheless the two actions are homotopic [11]. Via the functorality, the analogous considerations hold for the complex $T_{y} \circ \Delta_{\lambda}\left(F .\left(M_{\min }\right)\right)$ with respect to the left $\mathfrak{l}$ action and the induced $L_{0}$ action.

According to the comparison theorem in [2, Theorem 7] the homology groups $h_{p}\left(T_{y} \circ \Delta_{\lambda}\left(F .\left(M_{\min }\right)\right)\right)$ have Hausdorff topologies and the morphism $M \rightarrow M_{\text {min }}$ defines an $L_{0}$-equivariant isomorphism

$$
h_{p}\left(T_{y} \circ \Delta_{\lambda}(F \cdot(M))\right)_{\min } \simeq h_{p}\left(T_{y} \circ \Delta_{\lambda}\left(F \cdot\left(M_{\min }\right)\right)\right) .
$$

Thus the desired result follows immediately from Proposition 1.

\section{A proof of the comparison theorem.}

We can now complete the proof of Theorem 1 via some formal reductions and tensoring arguments.

For a moment, suppose that $M$ is a Harish-Chandra module with infinitesimal character $\sigma$. Observe that the structure of $H_{p}\left(\mathfrak{n}, M_{\min }\right)$ as a topological module for $L_{0}$ remains unchanged whether it is determined by the Hochschild resolution defined over the the algebra $U_{\sigma}$ or over $U(\mathfrak{g})$ since each of these algebras is $d n F$ with a countable basis and since continuous quasi-isomorphisms are good in the $d n F$ category [12, A.9 Corollary]. Using the Hochschild resolution with coefficients from $U_{\sigma}$ we obtain a complex

$$
H_{0}\left(\mathfrak{n}, F_{p}\left(M_{\min }\right)\right)=H_{0}\left(\mathfrak{n}, U_{\sigma}\right) \otimes^{p} U_{\sigma} \otimes M_{\min }
$$

that splits as a finite direct sum according to the splitting of $H_{0}\left(\mathfrak{n}, U_{\sigma}\right)$ into generalized $Z(\mathfrak{l})$ eigenspaces. Thus the $L_{0}$-module $H_{p}\left(\mathfrak{n}, M_{\min }\right)$ splits as a finite topological direct sum of its generalized $Z(\mathfrak{l})$-eigenspaces. As we saw in Lemma 2, in case $\sigma$ is a regular infinitesimal character, then $H_{p}(\mathfrak{n}, M)$ and $H_{p}\left(\mathfrak{n}, M_{\min }\right)$ are direct sums of regular $Z(\mathfrak{l})$-eigenspaces.

For an arbitrary Harish-Chandra module $M$ we use the Hochschild resolution defined over $U(\mathfrak{g})$ to specify the structure of $H_{p}\left(\mathfrak{n}, M_{\text {min }}\right)$ as a topological $L_{0}$ module. Nevertheless, in order to carry out the tensoring argument it seems we need to know that the generalized $Z(\mathfrak{l})$ eigenspaces occurring in $H_{p}\left(\mathfrak{n}, M_{\min }\right)$ can be computed as the homolgy groups of a complex of $d n F$ spaces. We continue with the notations of the previous section.

Proposition 2. Let $m$ be a positive integer and let $J_{\sigma}^{m} \subset Z(\mathfrak{g})$ be the ideal obtained by raising $J_{\sigma}$ to the $m$ th power. Let $U_{\sigma}^{m}$ be the corresponding 
quotient of $U(\mathfrak{g})$ by the ideal generated from $J_{\sigma}^{m}$. Then $U_{\sigma}^{m}$ is a free $U(\mathfrak{n})$ module.

Proof. The proof is essentially the same as when $m=1$ and $U_{\sigma}^{m}=U_{\sigma}$, [16].

Since a Harish-Chandra module $M$ and its minimal globalization are both $Z(\mathfrak{g})$-finite it follows that the homology groups $H_{p}(\mathfrak{n}, M)$ and $H_{p}\left(\mathfrak{n}, M_{\min }\right)$ are both finite direct sums of generalized $Z(\mathfrak{l})$ eigenspaces. If $V$ is an $\mathfrak{l}$ module (or alternatively a $\mathfrak{g}$ module) let $V_{(\lambda)}$ denote the generalized $Z(\mathfrak{l})$ (respectively $Z(\mathfrak{g})$ ) eigenspace corresponding to $\lambda \in \mathfrak{h}^{*}$. Then there is a natural isomorphism:

$$
H_{p}\left(\mathfrak{n}, M_{\min }\right)_{(\lambda)} \simeq H_{p}\left(\mathfrak{n}, M_{\min (\lambda)}\right)_{(\lambda)} .
$$

On the other hand, since $M_{(\lambda)}$ is finitely generated as a $U(\mathfrak{g})$-module, it follows by simple continuity considerations that there is an $m$ such that $M_{\min (\lambda)}$ is a module for $U_{\sigma}^{m}$. Using the the Hochschild resolution with coefficients in $U_{\sigma}^{m}$ we obtain a complex

$$
H_{0}\left(\mathfrak{n}, F_{p}\left(M_{\min (\lambda)}\right)\right)=H_{0}\left(\mathfrak{n}, U_{\sigma}^{m}\right) \otimes^{p} U_{\sigma}^{m} \otimes M_{\min (\lambda)}
$$

that splits as a finite direct sum according to the splitting of $H_{0}\left(\mathfrak{n}, U_{\sigma}^{m}\right)$ into generalized $Z(\mathfrak{l})$-eigenspaces. In particular, the generalized $Z(\mathfrak{l})$-eigenspaces in the $\mathfrak{n}$ homology groups $H_{p}\left(\mathfrak{n}, M_{\min }\right)_{(\lambda)}$ are the homology groups of a complex of $d n F$ spaces. Thus a standard long exact sequence argument coupled with facts about complexes of $d n F$ spaces [12, Proposition A.10] and the closed range condition for maps between minimal globalizations [12, Lemma 9.1] allows us to extend the result of Lemma 3 to arbitrary Harish-Chandra modules [13, Lemma 3.1]:

Lemma 4. Let $M$ be a Harish-Chandra module for $(\mathfrak{g}, K)$, fix $\lambda \in \mathfrak{h}^{*}$ and suppose the following two conditions hold whenever $M$ has an infinitesimal character:

(a) The induced topology on $H_{p}\left(\mathfrak{n}, M_{\min }\right)_{(\lambda)}$ is Hausdorff.

(b) The morphism $M \rightarrow M_{\min }$ determines an $L_{0}$-equivariant topological isomorphism $\left(H_{p}(\mathfrak{n}, M)_{(\lambda)}\right)_{\min } \simeq H_{p}\left(\mathfrak{n}, M_{\min }\right)_{(\lambda)}$

Then both conditions hold for all Harish-Chandra modules. Furthermore: If both conditions hold for every $\lambda \in \mathfrak{h}^{*}$ then Theorem 1 holds as well.

Let $\Sigma^{+}(\mathfrak{l}) \subset \Sigma^{+}$denote the set of positive roots for $\mathfrak{h}$ in $\mathfrak{l}$ and let $\Sigma(\mathfrak{n})=\Sigma^{+}-\Sigma^{+}(\mathfrak{l})$ denote the corresponding roots for $\mathfrak{h}$ in $\mathfrak{n}$. Given an infinitesimal character $\chi_{\lambda}$ for $\mathfrak{l}$ with corresponding parameter $\lambda \in \mathfrak{h}^{*}$ we define the length $n\left(\chi_{\lambda}\right)$ of the character [2, Section 9] to be the cardinality of 
$\{\alpha \in \Sigma(\mathfrak{n}) \mid \check{\alpha}(\lambda)$ is a positive integer $\}$. Observe that $n\left(\chi_{\lambda}\right)=0$ if and only if $\lambda$ is antidominant for $Y$. We also introduce the following terminology: $\lambda$ is called antidominant for $\mathfrak{l}$ provided $\check{\alpha}(\lambda)$ is not a positive integer for all $\alpha \in \Sigma^{+}(\mathfrak{l})$. Note that each $Z(\mathfrak{l})$ infinitesimal character has an $\mathfrak{l}$ antidominant parameter.

We now complete the argument by an induction in length, utilizing [18, Proposition 7.4.6] as well as [18, Proposition 7.4.7].

Lemma 5. Suppose $\lambda \in \mathfrak{h}^{*}$ is antidominant for $Y$ and that $M$ is a Harish-Chandra module for $(\mathfrak{g}, K)$. Otherwise make the same assumptions as Lemma 3. Then we obtain the conditions (a) and (b) of Lemma 4.

Proof. We may assume that $\lambda$ is antidominant. There is a finite dimensional irreducible $G_{0}$-module $F^{\mu}$ with lowest weight $\mu$ such that $\lambda+\mu$ is antidominant and regular. Let $F_{L_{0}}^{\mu}$ be the irreducible $L_{0}$-module of lowest weight $\mu$. Filtering $F^{\mu}$ by $\mathfrak{p}$ invariant subspaces with subquotients irreducible $L_{0^{-}}$ modules and arguing as in [18, Proposition 7.4.6] we obtain a commutative square with horizontal isomorphims:

$$
\begin{gathered}
H_{p}\left(\mathfrak{n}, M_{\min } \otimes F^{\mu}\right)_{(\lambda+\mu)} \simeq\left(H_{p}\left(\mathfrak{n}, M_{\min }\right)_{(\lambda)} \otimes F_{L_{0}}^{\mu}\right)_{(\lambda+\mu)} \\
\uparrow \\
\uparrow \\
H_{p}\left(\mathfrak{n}, M \otimes F^{\mu}\right)_{(\lambda+\mu)} \simeq\left(H_{p}(\mathfrak{n}, M)_{(\lambda)} \otimes F_{L_{0}}^{\mu}\right)_{(\lambda+\mu)} .
\end{gathered}
$$

Let $W=H_{p}(\mathfrak{n}, M)_{(\lambda)}$ and let $\Psi(W)=\left(W \otimes F_{L_{0}}^{\mu}\right)_{(\lambda+\mu)}$. Then it follows from Lemma 3 and Lemma 4 that the morphism $W \rightarrow H_{p}\left(\mathfrak{n}, M_{\min }\right)_{(\lambda)}$ determines a topological isomorphism: $\Psi(W)_{\min } \simeq \Psi\left(H_{p}\left(\mathfrak{n}, M_{\min }\right)_{(\lambda)}\right)$.

We utilize the following result on the translation functors:

Proposition 3. Let $\lambda \in \mathfrak{h}^{*}$ and suppose $\mu$ is the extremal weight of a finite dimensional irreducible $\mathfrak{l}$ module $F_{\mathfrak{l}}^{\mu}$. Let $U$ and $V$ be $\mathfrak{l}$ modules with generalized infinitesimal character $\chi_{\lambda}$ and view the translation functor

$$
\Psi(U)=\left(U \otimes F_{\mathfrak{l}}^{\mu}\right)_{(\lambda+\mu)}
$$

as defined on the category of $\mathfrak{l}$ modules with generalized infinitesimal character $\lambda$. Then the functor $\Psi$ is exact and there is a a surjection:

$$
\operatorname{Hom}_{U(\mathfrak{l})}(U, V) \rightarrow \operatorname{Hom}_{U(\mathfrak{l})}(\Psi(U), \Psi(V)) .
$$

Now suppose that $\lambda$ is an $\mathfrak{l}$ antidominant parameter and suppose in addition that for any $w \in W_{\mathfrak{l}}$ and for any $\nu$ a weight of $F_{\mathfrak{l}}^{\mu}$ that the equation $\lambda+\mu-\nu=w \lambda$ is satisfied if and only if $w \lambda=\lambda$ and $\mu=\nu$. Then $\Psi$ 
is faithful in the sense that $\Psi(U)=0$ if and only if $U=0$. In particular, $\operatorname{Hom}_{U(\mathfrak{l})}(U, V)$ is isomorphic to $\operatorname{Hom}_{U(\mathfrak{l})}(\Psi(U), \Psi(V))$.

Proof of Proposition 3. The exactness of $\Psi$ is clear, since $W \otimes F_{\mathfrak{l}}^{\mu}$ is $Z(\mathfrak{l})$ finite [18, Proposition 4.5.8 and Corollary 7.13]. On the other hand, the surjection of the Hom groups can be deduced directly from the adjointness formulas [18, Proposition 4.5.8]. Finally, the last claim can be easily deduced (for example) by using the geometric translation functors and the facts about algebraic localization on a full flag manifold [16]. In particular, one can easily deduce that the adjoint to $\Psi$ maps essentially onto the category of $\mathfrak{l}$ modules with $Z(\mathfrak{l})$ infinitesimal character $\lambda$.

Because the translation functors commute with the minimal globalization, to complete the proof of Lemma 5 , it suffices to see that $H_{p}\left(\mathfrak{n}, M_{\min }\right)_{(\lambda)}$ has a Hausdorff topology. But this follows directly, since the map obtained from the adjointness morphism:

$$
H_{p}\left(\mathfrak{n}, M_{\min }\right)_{(\lambda)} \rightarrow\left(\Psi(W)_{\min } \otimes F^{-\mu}\right)_{(\lambda)}
$$

is continuous and injective by Proposition 3 .

In order to complete the induction we need to know that there are enough finite dimensional irreducible $\mathfrak{l}$-modules. To obtain the weight $\mu$ that we need, we may have to pass to a finite covering group $\widetilde{G_{0}} \rightarrow G_{0}$, but this does not present a serious difficulty.

Lemma 6. Let $\lambda$ be an $\mathfrak{l}$ antidominant parameter for the $\mathfrak{l}$ infinitesimal character $\chi_{\lambda}$ and suppose that $n\left(\chi_{\lambda}\right)$ is positive. For any $\alpha \in \Sigma$ let $s_{\alpha} \in W$ denote the corresponding Weyl group reflection. Then there exists:

(i) A finite covering group $\widetilde{G_{0}} \rightarrow G_{0}$,

(ii) an extremal weight $\mu \in \mathfrak{h}^{*}$ for a finite dimensional irreducible representation $F^{\mu}$ of $\widetilde{G_{0}}$,

(iii) and an $\alpha \in \Sigma(\mathfrak{n})$

such that the following holds:

(1) $n\left(\chi_{\lambda+\mu}\right)$ and $n\left(\chi_{s_{\alpha}(\lambda)}\right)$ are both less than $n\left(\chi_{\lambda}\right)$.

(2) For $w \in W$ and $\nu$ a weight of $\mathfrak{h}$ in $F^{\mu}$ the equation:

$$
w \lambda+\nu=\lambda+\mu
$$

has a solution if and only if $w \lambda=\lambda$ and $\nu=\mu$ or else if $w \lambda=s_{\alpha} \lambda$ and $\nu=s_{\alpha} \mu$. 
Proof. This result is essentially shown in [16]. In fact, the desired result can be deduced directly from [2, Lemmas 13, 14 and 15] by utilizing the irreducible finite dimensional representation dual to the one utilized in $[2]$.

We now apply the long exact sequence of [18, Proposition 7.4.7] to the above data.

Lemma 7. Let $\lambda$ and $\mu$ be as in the previous lemma and suppose $M$ is a Harish-Chandra for $(\mathfrak{g}, K)$. Changing notations briefly, let $\widehat{M}$ denote the minimal globalization of $M$ and let $V=\left(M \otimes F^{\mu}\right)_{(\lambda+\mu)}$. Suppose that $\widetilde{L_{0}} \subset$ $\widetilde{G_{0}}$ is the real Levi factor associated to $\mathfrak{p}$ and let $F_{L_{0}}^{\mu}$ denote the irreducible $\widetilde{L_{0}}$ module with extremal weight $\mu$. Then there exists the following morphism of long exact sequences:

$$
\begin{gathered}
\rightarrow\left(H_{p}(\mathfrak{n}, \widehat{M})_{\left(s_{\alpha} \lambda\right)} \otimes F_{L_{0}}^{\mu}\right)_{(\lambda+\mu)} \rightarrow H_{p}(\mathfrak{n}, \widehat{V})_{(\lambda+\mu)} \rightarrow\left(H_{p}(\mathfrak{n}, \widehat{M})_{(\lambda)} \otimes F_{L_{0}}^{\mu}\right)_{(\lambda+\mu)} \rightarrow \\
\uparrow \\
\uparrow \\
\rightarrow\left(H_{p}(\mathfrak{n}, M)_{\left(s_{\alpha} \lambda\right)} \otimes F_{L_{0}}^{\mu}\right)_{(\lambda+\mu)} \rightarrow H_{p}(\mathfrak{n}, V)_{(\lambda+\mu)} \rightarrow\left(H_{p}(\mathfrak{n}, M)_{(\lambda)} \otimes F_{L_{0}}^{\mu}\right)_{(\lambda+\mu)} \rightarrow .
\end{gathered}
$$

To complete the proof of the comparison theorem we only need to see that $H_{p}\left(\mathfrak{n}, M_{\min }\right)_{(\lambda)}$ is the minimal globalization of $H_{p}(\mathfrak{n}, M)_{(\lambda)}$ for an arbitrary $\lambda \in \mathfrak{h}^{*}$. Using induction, the previous lemma and some considerations mentioned earlier, we see that $\left(H_{p}\left(\mathfrak{n}, M_{\min }\right)_{(\lambda)} \otimes F_{L_{0}}^{\mu}\right)_{(\lambda+\mu)}$ is the minimal globalization of $\left(H_{p}(\mathfrak{n}, M)_{(\lambda)} \otimes F_{L_{0}}^{\mu}\right)_{(\lambda+\mu)}$. Thus the desired result follows by Proposition 3.

\section{Cohomology and topological duality.}

We begin this last section by briefly recalling a few notions involved in defining $H_{p}\left(\mathfrak{n}, M_{\min }\right)=\operatorname{Tor}_{U(\mathfrak{n})}^{p}\left(\mathbb{C}, M_{\min }\right)$ as a topological module [12, Section 2]. A free $d n F$ module for $U(\mathfrak{n})$ is any module of the form $U(\mathfrak{n}) \otimes A$ where $A$ is a $d n F$ space. When $A$ is also an $\mathfrak{n}$-module then $A$ is a topological $d n F$ module precisely when the action map $U(\mathfrak{n}) \otimes A \rightarrow A$ is continuous. In this case the map $A \rightarrow U(\mathfrak{n}) \otimes A$ by $a \mapsto a \otimes 1$ defines a splitting of $U(\mathfrak{n}) \otimes A$ as a topological vector space.

Now suppose that $A$ and $B$ are $d n F$ modules and suppose that we have a continuous surjection $B \rightarrow A \rightarrow 0$ of modules which we also assume defines a splitting of $B$ as a topological vector space. In addition suppose that $F$ is a free $d n F$ module and that there is a continuous morphism $F \rightarrow A$ of $U(\mathfrak{n})$ 
modules. Then there exists a continuous morphism $F \rightarrow B$ of modules such that the diagram:

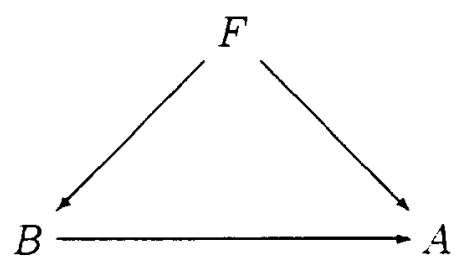

commutes. Thus an application of [12, Proposition A.8] shows that the structure of of $\operatorname{Tor}_{U(\mathfrak{n})}^{p}(\mathbb{C}, A)$ as a topological module is independent of the free resolution of $A$.

On the other hand one can resolve $\mathbb{C}$ by free $d n F$ modules. Then a standard argument, coupled with (for example) [12, Corollary A.11] shows that once again the same topological module is obtained. In particular, if $M$ is a Harish-Chandra module for $(\mathfrak{g}, K)$ and if $C .\left(\mathfrak{n}, M_{\min }\right)$ denotes the standard Koszul complex

$$
C_{p}\left(\mathfrak{n}, M_{\min }\right)=\bigwedge^{p} \mathfrak{n} \otimes M_{\min }
$$

then it follows from the work in the previous section that this complex has Hausdorff homology. Thus, using the standard complex to relate Lie algebra homolgy with Lie algebra cohomolgy [10, 2.17 and 2.18] and applying the results of the previous section, we can obtain a comparison theorem for the Lie algebra cohomolgy groups [4, Theorem 1.3]. That is: making the same assumptions as in Theorem 1 we obtain natural isomorphisms for the Lie algebra cohomology groups:

$$
H^{p}(\mathfrak{n}, M)_{\min } \simeq H^{p}\left(\mathfrak{n}, M_{\min }\right) .
$$

The standard complex also defines a natural duality relating the Lie algebra cohomolgy of the dual with the dual of the Lie algebra homolgy [10, 2.17 and 2.18]. Since the continuous dual defines an exact functor on $d n F$ spaces and since the standard complex has Hausdorff homology, this identification passes to continuous duals. Thus one can also express the content of Theorem 1 in terms of $\mathfrak{n}$-homologies (or $\mathfrak{n}$-cohomologies) of the maximal globalization [14], [17] of a Harish-Chandra module (although we remark that the comparison theorem is in fact false for a maximal globalization as was pointed out to us by H. Hecht). In particular, let $M^{\vee}$ denote the $K$ finite dual of $M$ and let $M_{\max }^{\prime}$ denote the continuous dual of the maximal globalization $M_{\max }$ of $M$. Then there is a natural isomorphism $M_{\max }^{\prime} \simeq M_{\min }^{\vee}$ 
[17]. Let $d$ be the dimension of $\mathfrak{n}$. Then it follows from the previous considerations that the $n$-homologies (as well as the $\mathfrak{n}$-cohomologies) of a maximal globalization provide maximal globalizations for the real Levi factor and that we can (for example) rephrase Theorem 1 in terms of the following Poincaré duality for Lie algebra cohomology [3, Proposition 4.4]:

$$
H^{p}\left(\mathfrak{n}, M_{\max }\right)^{\prime} \simeq H^{d-p}\left(\mathfrak{n}, M_{\min }^{\vee}\right) \otimes \bigwedge^{d} \mathfrak{n}
$$

\section{References}

[1] A. Beilinson and J. Bernstein, Localization de $\mathfrak{g}$ modules, C.R. Acad. Sci. Paris, 292 (1981), 15-18.

[2] T. Bratten, Realizing Representations on Generalized Flag Manifolds, preprint, to appear in Compositio Math., 1995.

[3] U. Bunke and M. Olbrich, Г-cohomology and the Selberg Zeta Function, preprint, Humboldt-Universitat zu Berlin, to appear in J. reine angew. Math., Dec. 1994.

[4] Cohomological properties of the canonical globalizations of Harish-Chandra modules, preprint, Humbolt-Universitat zu Berlin, August 1995.

[5] W. Casselman and M. Osborne, The $\mathfrak{n}$ cohomology of representations with an infinitesimal character, Compositio Math., 31 (1975), 219-227.

[6] J. Chang, Special K-types, tempered characters and the Beilinson-Bernstein realization, Duke Math. J., 56 (1988), 345-383.

[7] A. Deitmar, On some zeta functions attached to compact Shimura manifolds, preprint, Math. Inst. Im Neuenheimer Feld 288, 69126, Heidelberg, Germany, 1995.

[8] Harish-Chandra, Harmonic Analysis on real reductive groups I, J. Func. Anal., 19 (1975), 104-204.

[9] H. Hecht, D. Miličić, W. Schmid and J. Wolf, Localization and standard modules for semisimle Lie groups I: The duality theorem, Invent. Math., 90 (1987), 297-332.

[10] H. Hecht and W. Schmid, Characters, asymptotics and $\mathfrak{n}$-homology of HarishChandra modules, Acta mathematica, 151 (1983), 49-151.

[11] H. Hecht and J. Taylor, Some remarks on characters of semisimple Lie groups, preprint, Mathematics Department, University of Utah.

[12] H. Hecht and J. Taylor, Analytic localization of group representations, Advances in Math., 79(2) (1990), 139-212.

[13] H. Hecht and J. Taylor, A comparison theorem for $\mathfrak{n}$ homology, Composito Math., 86 (1993), 189-207.

[14] Masaki Kashiwara and Wilfred Schmid, Quasi-equivariant D-modules, equivariant derived category and representations of reductive Lie groups, Research anouncement, Research Institute for Mathematical Sciences, Kyoto University, 1994.

[15] T. Matsuki, Orbits of affine symmetric spaces under the action of minimal parabolic subgroups, J. Math. Soc. Japan, 31 (1979), 331-357.

[16] D. Miličić, Localization and Representation Theory of Reductive Lie Groups, text in preparation, Mathematics Department, University of Utah. 
[17] W. Schmid, Boundary value problems for group invariant differential equations, Proc. Cartan Symposium, Astérique, 1985.

[18] D. Vogan, Representations of Real Reductive Lie Groups, Progress in Math., 15, Birkhauser, 1981.

Received June 12, 1995 and revised April 15, 1997.

Universidad Nacional del Centro de la Provincia de Buenos Aires Campus Universitario, Paraje Arroyo Seco

7000 Tandil, Argentina 